\title{
Expression of Vitreoscilla hemoglobin enhances production of arachidonic acid and lipids in Mortierella alpina
}

\author{
Huidan Zhang ${ }^{1,3,4}$, Yingang Feng ${ }^{1,3}$, Qiu Cui ${ }^{1,2,3^{*}}$ and Xiaojin Song ${ }^{1,3^{*}}$ (D)
}

\begin{abstract}
Background: Arachidonic acid (ARA, C20:4, n-6), which belongs to the omega- 6 series of polyunsaturated fatty acids and has a variety of biological activities, is commercially produced in Mortierella alpina. Dissolved oxygen or oxygen utilization efficiency is a critical factor for Mortierella alpina growth and arachidonic acid production in large-scale fermentation. Overexpression of the Vitreoscilla hemoglobin gene is thought to significantly increase the oxygen utilization efficiency of the cells.

Results: An optimized Vitreoscilla hemoglobin ( $\mathrm{VHb}$ ) gene was introduced into Mortierella alpina via Agrobacterium tumefaciens-mediated transformation. Compared with the parent strain, the VHb-expressing strain, termed VHb-20, grew faster under both limiting and non-limiting oxygen conditions and exhibited dramatic changes in cell morphology. Furthermore, VHb-20 produced 4- and 8-fold higher total lipid and ARA yields than those of the wild-type strain under a microaerobic environment. Furthermore, ARA production of VHb-20 was also 1.6-fold higher than that of the wild type under normal conditions. The results demonstrated that DO utilization was significantly increased by expressing the $\mathrm{VHb}$ gene in Mortierella alpina.
\end{abstract}

Conclusions: The expression of VHb enhances ARA and lipid production under both lower and normal dissolved oxygen conditions. This study provides a novel strategy and an engineered strain for the cost-efficient production of ARA.

Keywords: Arachidonic acid, Mortierella Alpina, Hemoglobin, Dissolved oxygen, Fermentation

\section{Background}

Arachidonic acid (ARA, 5,8,11,14-cis-eicosatetraenoic acid), belonging to the omega- 6 series of polyunsaturated fatty acids (PUFAs), is not only a structural component of the cell membrane but also the biogenetic precursor of prostaglandins, leukotrienes, thromboxanes, and other eicosanoid hormones $[1,2]$. Furthermore, ARA is widely used in health food, pharmacology, agriculture, cosmetics and other industries [3, 4]. Mortierella alpina, which has a high ARA content, is considered one of the best ARA-producing strains and has been used in industrial applications [5].

\footnotetext{
* Correspondence: cuiqiu@qibebt.ac.cn; songxj@qibebt.ac.cn

'Shandong Provincial Key Laboratory of Energy Genetics, Qingdao Institute of Bioenergy and Bioprocess Technology, Chinese Academy of Sciences, No.189 Songling Road, Laoshan District, Qingdao, Shandong Province 266101, China

Full list of author information is available at the end of the article
}

The growth of $M$. alpina is closely related to the dissolved oxygen, especially in high cell density fermentation. The fast growth of the mycelium can result in an apparent increase in broth viscosity. The mycelium can agglomerate easily and further decrease the efficiency of oxygen mass transfer [6, 7]. Therefore, improving the concentration of dissolved oxygen in the fermentation process or increasing the utilization of dissolved oxygen is key to improving the yield of ARA.

At present, there are various methods to improve the oxygen supply, for example, by increasing the stirring speed or ventilation, or by adding surfactants to increase the concentration of dissolved oxygen in the fermentation. However, these methods require either special equipment or high energy consumption $[8,9]$. It has been shown that the expression of the bacterial (Vitreoscilla) hemoglobin $(\mathrm{VHb})$ is an effective method to solve the problem of oxygen supply in the fermentation process because $\mathrm{VHb}$ can 
improve the efficiency of intracellular oxygen transport $[10,11]$. VHb is an oxygen-binding protein produced by Vitreoscilla, which allows the Vitreoscilla (aerobic bacteria) to survive under limited oxygen conditions. Khosla and Bailey first cloned the vgb gene and successfully expressed it in E. coli [12].They demonstrated that the expression of $\mathrm{VHb}$ could promote cell growth, increase protein synthesis capacity under limited oxygen conditions $[13,14]$. According to the characteristics of $\mathrm{VHb}$, it has a good application, which has been successfully engineered into different bacteria, fungi, as well as some plants and animals, to improve cell growth and to increase the expression of exogenous protein and metabolite production $[15,16]$.

The metabolic engineering method also has been applied to improve the ARA production. For example, Hao et al. [17] studied overexpression of ME2 and G6PD2 in M. alpina, increasing the supply of intracellular NADPH, with a 1.7-fold increase in total fatty acid and a 1.5-fold increase in arachidonic acid (ARA) content. In 2005, Takeno et al. [18] improved of the fatty acid composition of $M$. alpina 1S-4, through RNA interference with $\Delta 12$ desaturase gene expression. But ARA biosynthesis is multi-step, subject to multiple factors. The modification of one or two genes is not easy to produce transformants with the desired trait. Overexpression of $\mathrm{VHb}$ could increase the intensity of respiratory chain and increase the supply of intracellular ATP, which may affect the expression of intracellular multi-gene expression and promote the formation of ARA.

To the best of our knowledge, $\mathrm{VHb}$ has not been used in M. alpina to date. In this study, we investigate the effect of $\mathrm{VHb}$ on growth and ARA production in Mortierella alpina and explored the mechanism of $\mathrm{VHb}$ in ARA synthesis.

\section{Methods}

\section{Strains, plasmids and growth conditions}

Wild-type Mortierella alpina ATCC 32222 was obtained from the American Type Culture Collection (ATCC, Manassas, VA, USA). The Agrobacterium tumefaciens strain $\mathrm{C} 58 \mathrm{C} 1$ and plasmid pBIG4MRHrev were gifts from Yasuyuki Kubo (Kyoto Prefectural University, Japan). The strains, plasmids and primers used in this study are listed in Additional file 1: Table S1 and Table S2, respectively. The composition of the LB-Mg agar, minimal medium $(\mathrm{MM})$ and inducing medium (IM), which were used for the culture, transformation and infection of Agrobacterium tumefaciens, respectively, have been described previously [19]. GY medium (20 g L ${ }^{-1}$ glucose, $10 \mathrm{~g} \mathrm{~L}^{-1}$ yeast extract and $20 \mathrm{~g} \mathrm{~L}^{-1}$ agar) was used for the transformation and screening of Mortierella alpina. M. alpina strains were grown on PDA medium (potatoes $200 \mathrm{~g} \mathrm{~L}^{-1}$, glucose $20 \mathrm{~g}$ $\mathrm{L}^{-1}$, and agar $20 \mathrm{~g} \mathrm{~L}^{-1}$ ) slants at $25{ }^{\circ} \mathrm{C}$. The seed culture medium contained $30 \mathrm{~g} \mathrm{~L}^{-1}$ glucose, $6 \mathrm{~g} \mathrm{~L}^{-1}$ yeast extract, $3 \mathrm{~g} \mathrm{~L}^{-1} \quad \mathrm{KH}_{2} \mathrm{PO}_{4}, 2.8 \mathrm{~g} \mathrm{~L}^{-1} \mathrm{NaNO}_{3}$, and $0.5 \mathrm{~g} \mathrm{~L}^{-1}$ $\mathrm{MgSO}_{4} \cdot 7 \mathrm{H}_{2} \mathrm{O}$. The medium for shake-flask culture contained $80 \mathrm{~g} \mathrm{~L}^{-1}$ glucose, $11 \mathrm{~g} \mathrm{~L}^{-1}$ yeast extract, $3.8 \mathrm{~g} \mathrm{~L}^{-1}$ $\mathrm{KH}_{2} \mathrm{PO}_{4}, 3.5 \mathrm{~g} \mathrm{~L}^{-1} \mathrm{NaNO}_{3}$, and $0.5 \mathrm{~g} \mathrm{~L}^{-1} \mathrm{MgSO}_{4} \cdot 7 \mathrm{H}_{2} \mathrm{O}$. The medium for bioreactor fermentation contained $30 \mathrm{~g} \mathrm{~L}^{-1}$ glucose, $20 \mathrm{~g} \mathrm{~L}^{-1}$ yeast extract, $4 \mathrm{~g} \mathrm{~L}^{-1}$ $\mathrm{KH}_{2} \mathrm{PO}_{4}, \quad 3.8 \quad \mathrm{~g} \quad \mathrm{~L}^{-1} \mathrm{NaNO}_{3}$, and $0.6 \mathrm{~g} \mathrm{~L}^{-1}$ $\mathrm{MgSO}_{4} \cdot 7 \mathrm{H}_{2} \mathrm{O}$. The Escherichia coli strains were grown in $\mathrm{LB}$ medium $\left(10 \mathrm{~g} \mathrm{~L}^{-1}\right.$ tryptone, $5 \mathrm{~g} \mathrm{~L}^{-1}$ yeast extract, and $\left.10 \mathrm{~g} \mathrm{~L}^{-1} \mathrm{NaCl}\right)$ at $37^{\circ} \mathrm{C}$.

\section{Codon analysis and optimization}

The codon preference table was found by analyzing the codon preference of the gene of $M$. alpina by the online software Codon Usage Database (http://www.kazusa.or.jp/codon/). The $v g b$ gene (GenBank accession no.M30794.1) of Vitreoscilla was optimized by software Optimizer (http://genomes.urv.cat/OPTIMIZER/) based on the codon preference table. The codon-optimized nucleotide sequence of $v g b$ was synthesized by GenScript (Nanjing) Co., Ltd. (Jiangsu, China).

\section{Vector construction}

The hygromycin $B$ phosphotransferase gene $(\mathrm{HPH})$ expression cassette containing the hisH4.1 promoter, the hpt resistance gene and the trpC terminator was amplified with primer pair P1/T1 from the vector pD4 [20]. This HPH expression cassette was inserted into PMD19-T to yield the plasmid pMD19T-HPH. The 18S sequence, which was amplified from M. alpina ATCC 32222 cDNA with primer pair $18 \mathrm{~S}-\mathrm{F} / 18 \mathrm{~S}-\mathrm{R}$, was ligated to the pMD19T-HPH vector, and the resulting construct was named pMD19T-HPH-18S. The carboxin resistance gene (CBXB) [21] was amplified with the primer pair CBXB-F / CBXB-R and used to replace the $h p t$ gene, thus forming plasmid pMD19T-CBXB-18S. The CBXB expression cassette was amplified from plasmid pMD19T-CBXB-18S with primer pair P2/T2, digested with restriction endonucleases ClaI and EcoRI, and then ligated to T-DNA binary vector pBIG4MRHrev. The resulting plasmid was named pBIG-CBXB. The HisH4.1 promoter and trpC terminator were first amplified with primer sets P3-F/P3-R and T4-F/ T4- $\mathrm{R}$ from the vector $\mathrm{pBIG-CBXB}$, thus yielding fragments of $1.1 \mathrm{~kb}$ and $0.7 \mathrm{~kb}$, respectively.

The optimized $v g b$ sequence was inserted into pUC57 to create the plasmid pUC57-vgb (Additional file 1: Table S1). The primer pair VHb-F/VHb-R was used to amplify the optimized $v g b$ gene from the vector pUC57-vgb, forming a $441 \mathrm{bp}$ fragment. The $v g b$ gene cassette containing the hisH4.1 promoter, the $v g b$ gene and the trpC terminator was assembled by overlap PCR. The obtained $2.1 \mathrm{~kb} v g b$ gene cassette was digested with EcoRI and SmaI and then ligated to 
pBIG-CBXB, thus forming the plasmid pBIG-CBXB$\mathrm{VHb}$ (Additional file 1: Figure S2).

\section{Agrobacterium tumefaciens-mediated transformation of $M$. alpina}

The transformation method was optimized on the basis of previously described Agrobacterium tumefaciens-mediated transformation (ATMT) methods [19, 22]. $M$. alpina spores were collected from cultures growing on PDA agar medium, and the suspension was filtered through three layers of lens paper. A. tumefaciens C58C1 was electrotransformed with the plasmid pBIG$\mathrm{CBXB}-\mathrm{VHb}$. The positive transformants were confirmed by $\mathrm{PCR}$, and then a single colony containing plasmid pBIG-CBXB-VHb was selected to grow overnight in $5 \mathrm{~mL}$ of liquid LB-Mg medium containing $50 \mu \mathrm{g} \mathrm{mL}$ kanamycin and $50 \mu \mathrm{g} \mathrm{mL} \mathrm{m}^{-1}$ rifampin at $28{ }^{\circ} \mathrm{C}$ and $200 \mathrm{rpm}$. The bacterial cells were collected by centrifugation at $5000 \mathrm{rpm}$ for $3 \mathrm{~min}$ at room temperature and washed once with fresh IM, and then fresh IM was used to adjust the OD to $0.2-0.3$. After being incubated at $28{ }^{\circ} \mathrm{C}$ until an OD660 of 0.8 was reached, $100 \mu \mathrm{L}$ of the Agrobacterium culture was mixed with $100 \mu \mathrm{L}$ of M. alpina spore suspension $\left(10^{8} \mathrm{~mL}^{-1}\right)$ and then spread onto cellophane membranes, placed on co-cultivation medium (IM with $1.5 \%$ agar) and incubated at $23{ }^{\circ} \mathrm{C}$ for 2 days. After cocultivation, the cellophane membranes were transferred to GY plates containing $150 \mu \mathrm{g} \mathrm{mL}{ }^{-1}$ carboxin, $50 \mu \mathrm{g} \mathrm{mL}$ spectinomycin and $50 \mu \mathrm{g} \mathrm{mL}^{-1}$ cefotaxime to inhibit the growth of Agrobacterium. The plates were incubated at $25{ }^{\circ} \mathrm{C}$ until CBXB-resistant colonies became visible. The transformed candidates were transferred to GY agar plates containing $150 \mu \mathrm{g} \mathrm{mL}{ }^{-1}$ carboxin. To obtain stable transformants, this procedure was repeated three times, and all experiments were conducted in triplicate.

\section{PCR verification of the transformants}

PCR was used to identify whether the mycelium was successfully transformed. Genomic DNA of M. alpina strains was prepared by a method described previously [23]. The genomic DNAs of the transformants were used as PCR templates to confirm the integration of the plasmid pBIG-CBXB-VHb by using the $v g b$ gene specific primers VHb-F and VHb-R (Additional file 1: Table S2). Wild type $M$. alpina and the plasmid pBIG-CBXB-VHb were used as negative and positive PCR controls, respectively. The PCR products were run on a $1.0 \%$ agarose gel and stained with ethidium bromide.

\section{Analytical methods for dry mycelial weight, total lipid and arachidonic acid production \\ Cell dry weight determination}

In total, $50 \mathrm{~mL}$ cell suspensions were harvested by filtration at room temperature. The cell pellet was washed twice with distilled water and dried to constant weight at $50{ }^{\circ} \mathrm{C}$. The dry cell weight was determined by weight analysis and represented by dry cell weight (DCW).

\section{Lipid extraction and fatty acid composition analysis}

Fatty acid extraction and methylation were carried out according to the previously described methods [24]. Total lipids were extracted by using approximately $100 \mathrm{mg}$ of mycelia (dry weight). Fatty acid methyl esters (FAMEs) were obtained by reacting the lipids at $85{ }^{\circ} \mathrm{C}$ for $2.5 \mathrm{~h}$ in the presence of $2 \%$ sulfuric acid/methanol $(v / v)$. FAMEs were extracted in hexane and analyzed by gas chromatography (Agilent Technologies, 7890B) with an HP-INNOWAX capillary column $(30 \mathrm{~m}$ by $0.25 \mathrm{~mm}$, $0.25 \mu \mathrm{m}$ film thickness). The oven temperature was set at $100{ }^{\circ} \mathrm{C}$ for $1 \mathrm{~min}$, was raised to $250{ }^{\circ} \mathrm{C}$ at a rate of $15^{\circ} \mathrm{C}$ per minute and then was held at $250{ }^{\circ} \mathrm{C}$ for $5 \mathrm{~min}$. The split ratio was 1:19, and the carrier gas was nitrogen. The injection volume was $1 \mu \mathrm{L}$. Peak detection used a flame ionization detector (FID). The temperature of the flame ionization port and injection port was $280^{\circ} \mathrm{C}$.

\section{Shake-flask culture and bioreactor scale fermentation}

To study the effect of $\mathrm{VHb}$ on M. alpina, especially under the oxygen-restricted conditions, we compared the DCW, total lipid content, and arachidonic acid production between the transformant $\mathrm{VHb}-20$ and the wildtype strain in shake flasks and a bioreactor under $\mathrm{dO}_{2}$ limiting and non-limiting conditions. First, the VHb-20 transformants and wild type were inoculated into six $250 \mathrm{~mL}$ shake flasks with baffles. Three shake flasks contained $50 \mathrm{~mL}$ medium (normal, dO2 non-limiting condition), and three contained $150 \mathrm{~mL}$ medium (dO2 restriction). They were shaken at $25{ }^{\circ} \mathrm{C}$ and $200 \mathrm{rpm}$ for 7 days. Meanwhile, bioreactor cultures were grown in 5-L Biostat B plus bioreactors (Sartorius Stedim Biotech, Germany) with $3 \mathrm{~L}$ of medium. The rate of ventilation and agitation during growth in the bioreactor was set to non-limiting oxygen $(1.7 \mathrm{vvm}, 200 \mathrm{rpm})$ and limiting oxygen $(0.8 \mathrm{vvm}, 100 \mathrm{rpm})$ conditions. The transformant and wild-type cultures were grown for 7 days at $25^{\circ} \mathrm{C}, \mathrm{pH} 6.5$. The glucose concentration was maintained at approximately $20 \mathrm{~g} \mathrm{~L}^{-1}$ by feeding the $80 \%(\mathrm{w} / \mathrm{v}$ ) glucose solution, which was measured with a SBA-40D Biosensor (Institute of Biology, Shandong Academy of Sciences, China). Twenty milliliter samples were taken at $24 \mathrm{~h}$ intervals in all experiments. The entire fermentation process used bioprocess-automated software for monitoring and control.

\section{Quantitative real-time PCR (qRT-PCR) validation}

The expression levels of some desaturase genes, such as $\Delta 9, \Delta 12, \Delta 6$, and $\Delta 5$ desaturase, were further quantified by real-time PCR to validate the effects of $\mathrm{VHb}$ 
expression on the biosynthesis of unsaturated fatty acids. The primer sequences (Additional file 1: Table S3) were designed according to the genome sequencing data (NCBI BioProjects PRJNA41211). Total RNA was extracted using a thermo Scientific GeneJET RNA Purification kit (No. K0731). After DNA degradation by DNase, $2 \mu \mathrm{g}$ of total RNA was reverse transcribed using a TIANScript reverse transcription Kit (Tiangen) in a $20 \mu \mathrm{l}$ reaction volume.

qRT-PCR was performed in a LightCycler R480 Realtime Detection System (Roche). A melting curve analysis of the amplification products was performed to confirm the specificity of amplification. To quantify the transcription of each gene, the copy number was determined by generating a standard curve by using a serial 10 -fold dilution of the targeted PCR product inserted into the $\mathrm{pMD}^{\mathrm{ma}}$ 19-T vector (TaKaRa Bio Group). For sample normalization, 18S rRNA was used as an internal standard. All the reactions were performed in triplicate, and the data were normalized by using the average values for the internal standard.

\section{Calculation and statistical analysis}

All results are given as the mean \pm standard error of the mean (SEM), and significant differences $(P<0.05$, $P<0.01)$ between means were compared by the T-test.

\section{Results}

\section{The stabilized expression of $\mathrm{VHb}$ in M. alpina}

To express VHb efficiently in $M$. alpina, we designed and optimized the $v g b$ gene according to the codon preference in $M$. alpina. The optimized $v g b$ gene sequence is shown in Additional file 1: Figure S1.

We first tested the sensitivity of M. alpina ATCC 32222 to carboxin and another series of antibiotics (Additional file 1: Table S4). The strain did not grow in the presence of $150 \mu \mathrm{g} \mathrm{mL} \mathrm{L}^{-1}$ carboxin but showed high resistance to other antibiotics. Therefore, the carboxin resistance gene $(C B X B)$ was used as the selection marker $[21,25]$ at a screening concentration of $150 \mu \mathrm{g} \mathrm{mL}^{-1}$.

The plasmid pBIG-CBXB-VHb was transformed into M. alpina ATCC 32222 by ATMT. All clones were verified by PCR using genomic DNA as templates. To select the high-yield ARA strain, 14 correct clones verified by PCR were randomly selected to check ARA production in limiting and non-limiting oxygen conditions. After 7 $\mathrm{d}$ of cultivation, a positive clone exhibiting the highest ARA production (VHb-20) was identified. Thus, VHb-20 strain was chosen for all further experiments. The $v g b$ gene expression cassette inserted in clone VHb-20 was confirmed by genomic PCR amplification (see Fig. 1a and b).

To confirm the functional expression of $\mathrm{VHb}$ in $\mathrm{VHb}-$ 20 , the carbon monoxide (CO) difference spectra were measured, and the wild-type strain was used as a control.
VHb-20 showed a typical $\mathrm{VHb}$ CO-binding absorption peak at $419 \mathrm{~nm}$, whereas this absorption peak was not observed in the control (Fig. 1c). This result indicated that the $\mathrm{VHb}$ expressed in $\mathrm{VHb}-20$ was biologically active. Afterwards, VHb-20 was continuously inoculated and cultivated on carboxin-free PDA solid medium, and the integrated $v g b$ gene in the genome was monitored by PCR. The result indicated that the recombinant strain was genetically stable after 5 sub-cultivation cycles.

\section{Effects of VHb expression on the growth and ARA production of $M$. alpina}

Both VHb-20 and the wild-type strain were cultivated in $250 \mathrm{~mL}$ shake flasks under limiting and non-limiting oxygen conditions, and their growth patterns and ARA production were investigated. The results showed that the VHb-20 strain grew faster than the wild-type strain and formed more homogeneous mycelial pellets under both conditions. In addition, the hyphae of M. alpina ATCC 32222 were looser.

Under aerobic conditions (cultivated in a $250-\mathrm{mL}$ baffled flask containing $50 \mathrm{~mL}$ of medium at $25{ }^{\circ} \mathrm{C}$ and $200 \mathrm{rpm}$ ), the glucose consumption rates and dry weight of VHb-20 and wild-type strains were not obviously different after cultivation for 7 days (Fig. 2a and b), but the total lipid yield of strain $\mathrm{VHb}-20\left(7.97 \mathrm{~g} \mathrm{~L}^{-1}\right)$ was 3.15 fold higher than that of the wild-type strain $\left(2.53 \mathrm{~g} \mathrm{~L}^{-1}\right.$; Fig. 2c). In addition, the ARA production of the strain VHb-20 was $3.62 \mathrm{~g} \mathrm{~L}^{-1}$, which was 5.10 times higher than that of the wild-type strain $\left(0.71 \mathrm{~g} \mathrm{~L}^{-1}\right.$; Fig. 2d). To obtain a microaerobic condition with a lower level of dissolved oxygen, the liquid medium in the flask was increased from $50 \mathrm{~mL}$ to $150 \mathrm{~mL}$. Under these conditions, the VHb-20 strain had a higher glucose consumption rate and growth rate compared with that of the control strain (Fig. 2a and b), and lipid and ARA production of VHb-20 was also higher by 4.03 folds $\left(4.64 \mathrm{~g} \mathrm{~L}^{-1}\right.$ vs. $\left.1.15 \mathrm{~g} \mathrm{~L}^{-1}\right)$ and 8.36 folds $\left(1.84 \mathrm{~g} \mathrm{~L}^{-1}\right.$ vs. $\left.0.22 \mathrm{~g} \mathrm{~L}^{-1}\right)$, respectively (Fig. 2c, and d).

\section{Effect of $\mathrm{VHb}$ expression on the composition of fatty acids in M. alpina}

The concentration of dissolved oxygen affects not only cell growth but also the synthesis of fatty acids. Therefore, we further compared the fatty acid composition and content of $\mathrm{VHb}-20$ and the wild-type strain under aerobic or microaerobic conditions. VHb-20 and the wild-type strain produced similar types of fatty acids (Fig. 3), but the proportion of various fatty acids in the lipids had changed significantly (Table 1).The results showed that the proportion of palmitic acid (C16: 0), stearic acid (C18: 0) and oleic acid (C18: 1$)$ in the total fatty acids of wild 


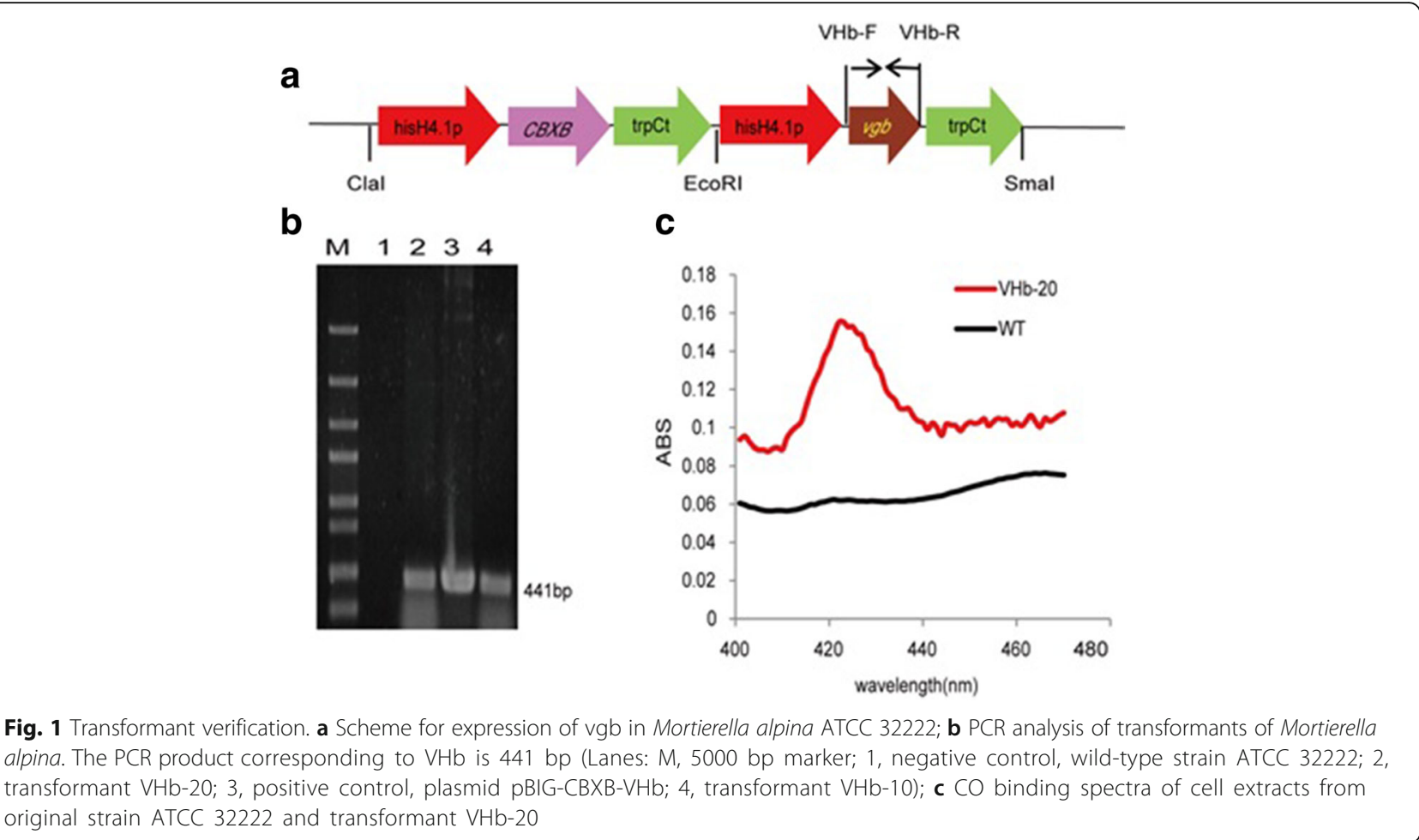

type strains was $15.82 \%, 17.98 \%$ and $17.58 \%$, significantly higher than other fatty acids. Especially in the limited oxygen condition, the accumulation of oleic acid (C18: 1) is very huge, the content in the total fatty acids as high as $39.61 \%$, resulting in ARA content was only $19.27 \%$. However, the contents of palmitic acid (C16: 0) and oleic acid (C18: 1) in VHb20 strain were significantly decreased, and the ARA content was increased to $45.68 \%$ and $38.51 \%$, under the aerobic or microaerobic conditions respectively, which were nearly 1.7 folds and 2.0 folds as high as that of the control.

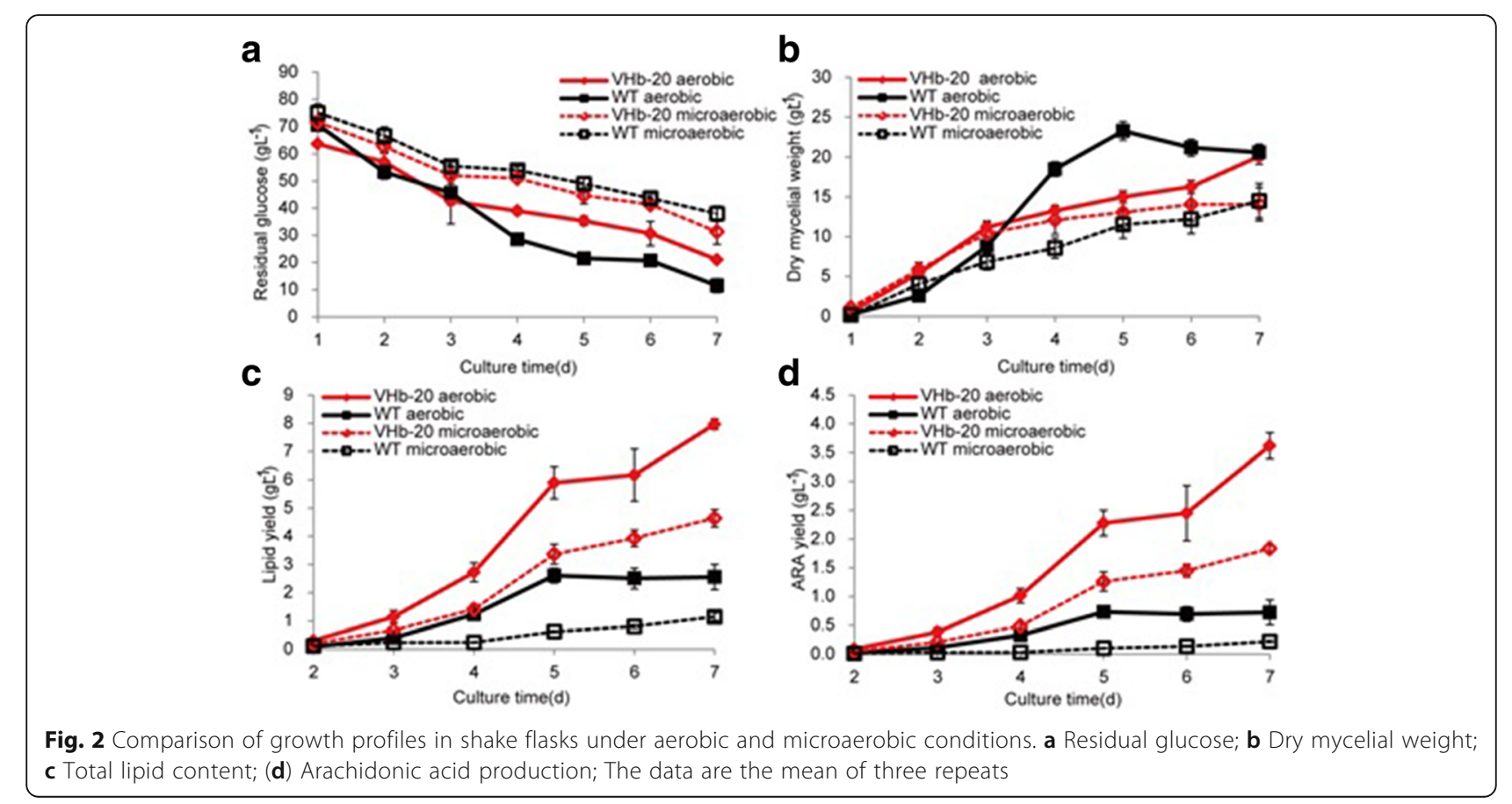




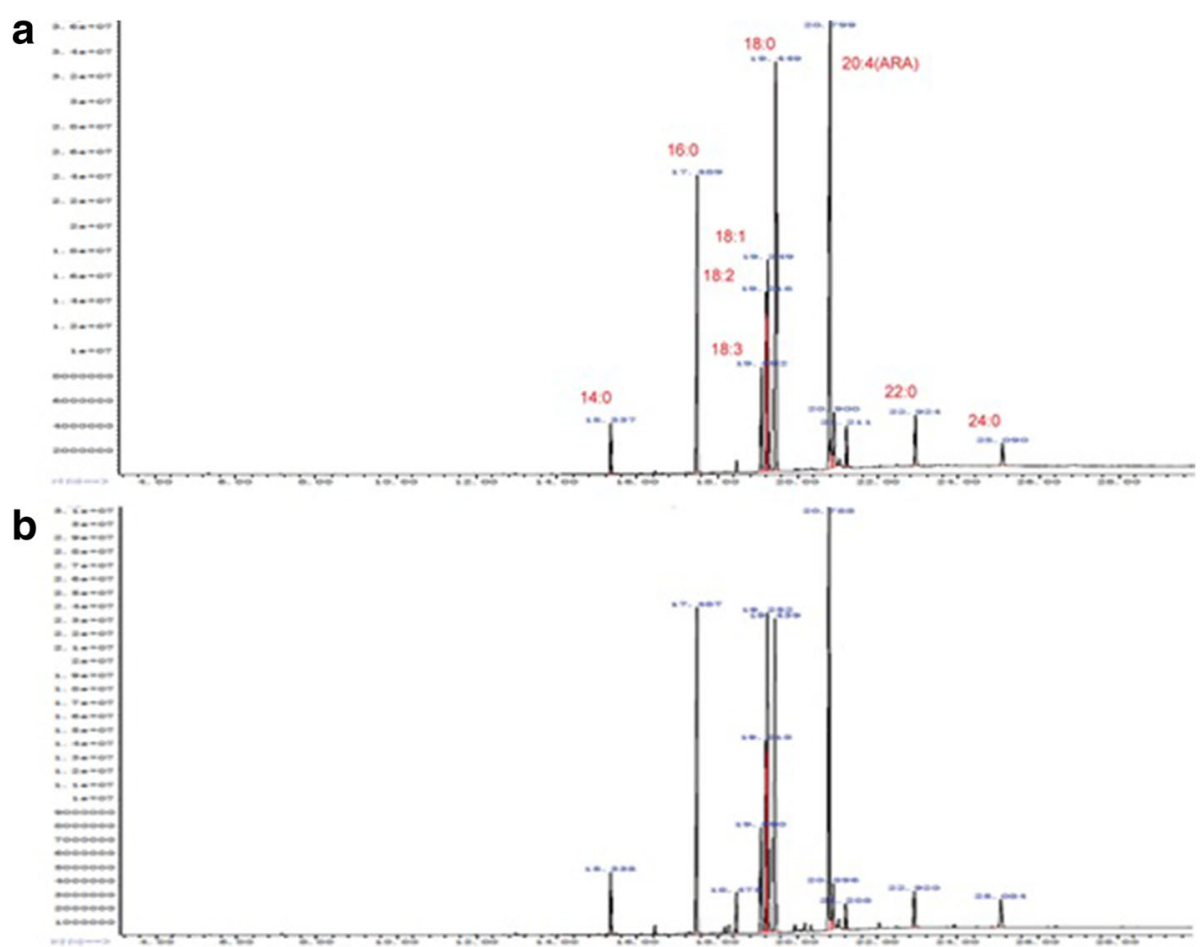

Fig. 3 GC-MS analysis of fatty acids composition from wild type and VHb-20. a VHb-20 in shake flasks with free air diffusion; b Wild type in shake flasks with free air diffusion

\section{Effects of $\mathrm{VHb}$ expression on the expression of desaturase genes}

The biosynthesis of unsaturated fatty acids in cell is catalyzed by desaturases, which require oxygen as a substrate [3]. Therefore, the expression of desaturase genes, including $\Delta 9$ desaturase, $\Delta 12$ desaturase, $\Delta 6$ desaturase, $\Delta 5$ desaturase, was investigated to verify the effects of $\mathrm{VHb}$ expression on the biosynthesis of unsaturated fatty acids at the molecular level. All the expression levels of these desaturase genes were higher in VHb-20, as compared with the wild type strain, in both aerobic and microaerobic conditions (Fig. 4a). From the results, $\Delta 9$ desaturase, which is the first enzyme in unsaturated fatty acid biosynthesis, was most sensitive to the expression of $\mathrm{VHb}$, which resulted 3.2 and 4.2-fold higher expression of the $\Delta 9$ desaturase gene under aerobic and microaerobic conditions, respectively. A lesser effect was observed in the increased expression of $\Delta 12$ and $\Delta 6$ desaturase, which were only 1.4-fold higher in VHb-20, and 1.6-fold higher expression of $\Delta 5$ desaturase was observed under aerobic conditions. However, under microaerobic conditions, the expression level of $\Delta 12$ and $\Delta 6$ desaturase was increased by 2.3 and 8.7 times higher than that of the control strain. In agreement with the

Table 1 Fatty Acid Compositions of wild type and VHb-20 in shake flasks

\begin{tabular}{|c|c|c|c|c|}
\hline \multirow[t]{2}{*}{ Fatty acid } & \multicolumn{4}{|c|}{ Content (\% total fatty acids) } \\
\hline & WT(aerobic) & VHb-20(aerobic) & WT(microaerobic) & VHb-20(microaerobic) \\
\hline $14: 0$ & $3.22 \pm 0.14$ & $0.73 \pm 0.02^{* *}$ & $2.84 \pm 0.04$ & $1.46 \pm 0.17^{* *}$ \\
\hline $16: 0$ & $15.82 \pm 0.33$ & $9.72 \pm 0.36^{* *}$ & $13.35 \pm 0.10$ & $11.51 \pm 0.24^{* *}$ \\
\hline $18: 0$ & $17.98 \pm 0.49$ & $19.63 \pm 0.73^{* *}$ & $13.34 \pm 0.95$ & $23.19 \pm 0.48^{* *}$ \\
\hline $18: 1$ & $17.58 \pm 0.58$ & $6.92 \pm 0.55^{* *}$ & $39.61 \pm 2.26$ & $8.34 \pm 0.24^{* *}$ \\
\hline $18: 2$ & $7.43 \pm 0.44$ & $5.82 \pm 0.22^{* *}$ & $2.87 \pm 0.35$ & $5.94 \pm 0.56^{* *}$ \\
\hline $18: 3$ & $3.37 \pm 0.11$ & $3.74 \pm 0.08^{*}$ & $2.27 \pm 0.38$ & $3.71 \pm 0.22^{*}$ \\
\hline $20: 3$ & $3.43 \pm 0.06$ & $2.84 \pm 0.19^{*}$ & $3.71 \pm 0.08$ & $2.38 \pm 0.26^{* *}$ \\
\hline 20:4(ARA) & $27.30 \pm 0.77$ & $45.68 \pm 1.09^{* *}$ & $19.27 \pm 1.18$ & $38.51 \pm 0.44^{* *}$ \\
\hline
\end{tabular}



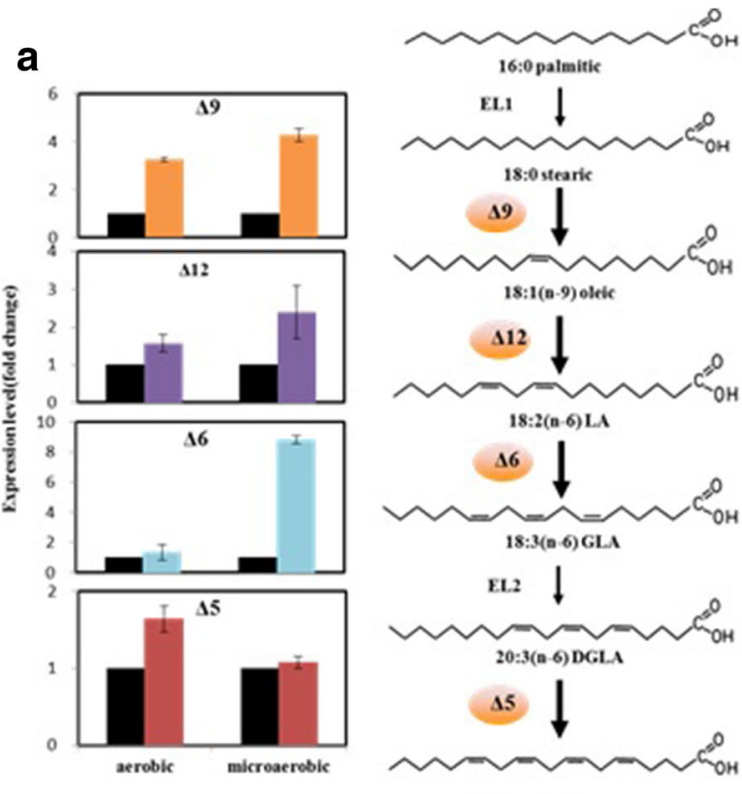

\section{b}
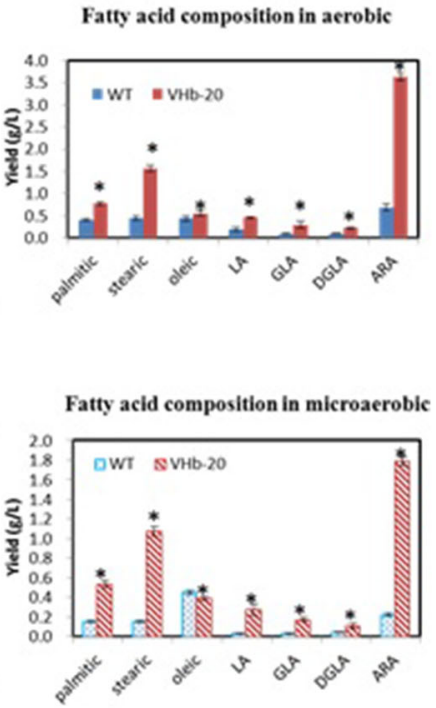

Fig. 4 The changes of the expression of desaturases ( $\Delta 9$ desaturase, $\Delta 12$ desaturase, $\Delta 6$ desaturase, $\Delta 5$ desaturase) and ARA production between the WT and VHb-20 strains under aerobic and microaerobic conditions. a Changes in the expression of desaturases, Black: WT; Colored: VHb-20 strain; $\mathbf{b}$ Changes in unsaturated fatty acid production $\left({ }^{*} p<0.05\right)$

differences in the levels of these desaturases, the biosynthesis of unsaturated fatty acids was augmented, and the ARA production of $\mathrm{VHb}-20$ was increased to $3.62 \mathrm{~g} \mathrm{~L}^{-1}$ and $1.84 \mathrm{~g} \mathrm{~L}^{-1}$ under the aerobic and microaerobic conditions (Fig. 4b), respectively.

\section{Confirmation of the effects of VHb expression on cell growth and ARA production by using bioreactor fermentation}

Bioreactor cultures were grown to confirm the influence of $\mathrm{VHb}$ expression on cell growth and lipid production under both non-limiting (1.7vvm, $200 \mathrm{rpm})$ and limiting $(0.8 \mathrm{vvm}, 100 \mathrm{rpm}) \mathrm{dO} 2$ conditions. From a morphological viewpoint (Fig. 5), VHb-20 grew faster and formed more uniform mycelial pellets than did the wildtype strain M. alpina ATCC 32222. In hypoxic conditions, the VHb-20 strain grew faster than the wild-type strain and reached the maximum biomass $(12.90 \%$ more than the wild type) sooner (Fig. 6a). In addition, the culture of the wild-type strain clumped heavily and became difficult to stir after 3 days of cultivation, whereas uniform mycelial pellets were observed in the VHb-20 culture, and the culture broth maintained good fluid properties through 7 days of fermentation.

In agreement with the results of the shake flask assay, $\mathrm{VHb}$ expression also enhanced lipid and ARA biosynthesis in the bioreactor experiments, especially under limiting oxygen conditions. There was almost no difference in the lipid content of the DCW of VHb-20 in the

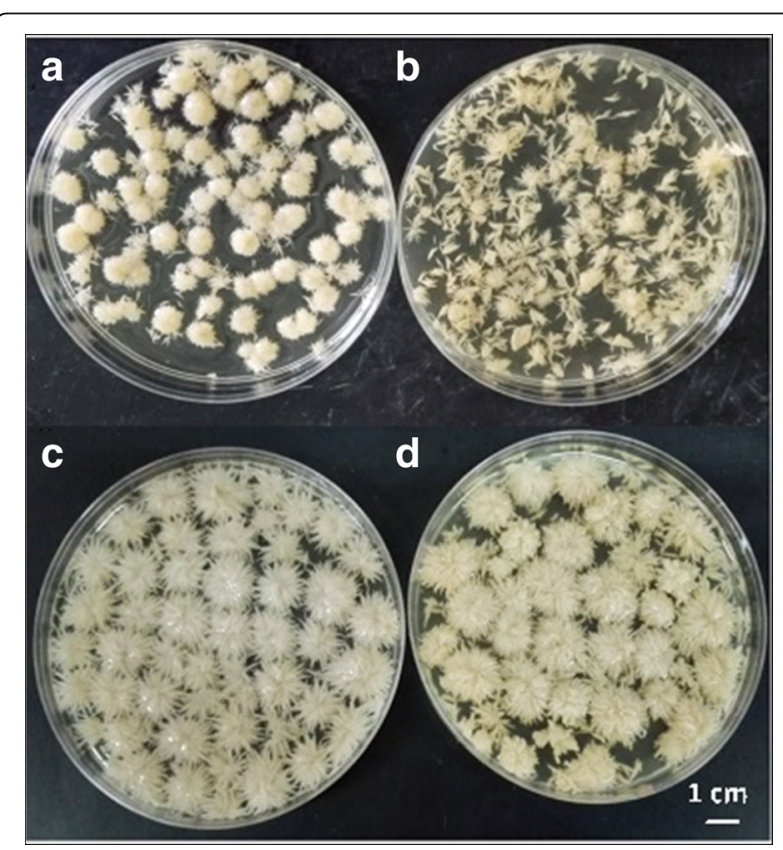

Fig. 5 Morphology of hyphae in $5 \mathrm{~L}$ fermenter. a VHb-20 transformant under aerobic conditions; $\mathbf{b}$ wild type under vaerobic conditions; $\mathbf{c}$ VHb-20 transformant under microaerobic conditions; $\mathbf{d}$ wild type under microaerobic conditions 

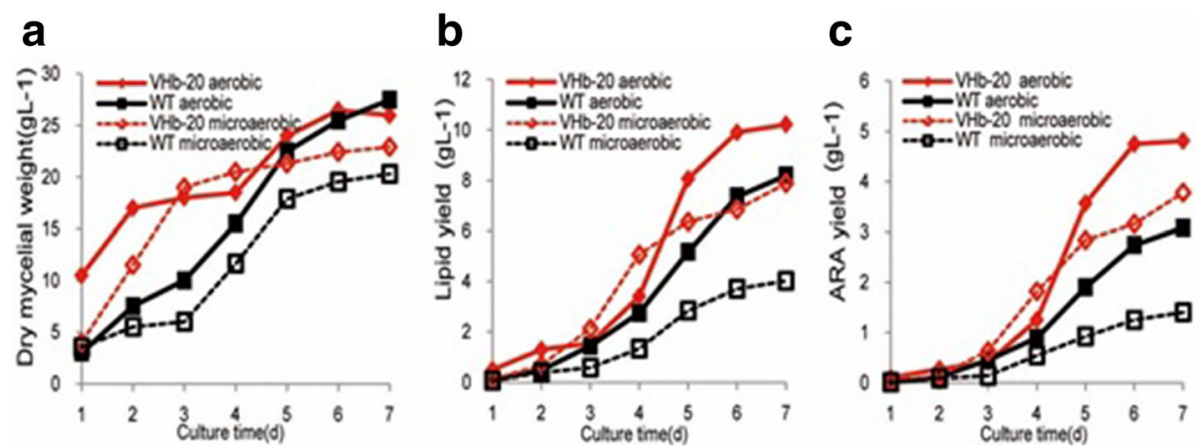

Fig. 6 Comparison of growth profiles in a 5-L fermenter under aerobic and microaerobic conditions. a Dry mycelial weight; b Total lipid content; c Arachidonic acid production

two conditions $(39.30 \%$ vs $34.40 \%$ for the normal and limiting oxygen conditions, respectively), but in the control, the lipid content was $34 \%$ lower in limiting oxygen conditions $(29.80 \%$ vs $19.80 \%)$ at the end of fermentation. The maximum lipid yield of VHb-20 strain was $10.21 \mathrm{~g} \mathrm{~L}^{-1}$ and $7.89 \mathrm{~g} \mathrm{~L}^{-1}$, which was nearly 1.25 and 1.96 fold higher than that of the original strain under the two aerobic conditions (Fig. 6b).

Under aerobic conditions, VHb-20 had a higher ARA yield by 1.56 -fold, from a value of $3.08 \mathrm{~g} \mathrm{~L}^{-1}$ to $4.81 \mathrm{~g} \mathrm{~L}^{-1}$, compared with the original strain M. alpina ATCC 32222. At the end of fermentation, the ARA yield of $\mathrm{VHb}-20$ was 2.70 times $\left(3.78 \mathrm{~g} \mathrm{~L}^{-1}\right.$ vs $1.40 \mathrm{~g} \mathrm{~L}^{-1}$ ) higher than that of the wild type under microaerobic conditions (Fig. 6c).

\section{Discussion}

ARA is an essential polyunsaturated fatty acid in human nutrition that has broad applications in many industries [26]. Although M. alpina is considered to be a good production strain for high level of ARA production, the low percentage of ARA in total lipids results in a production bottleneck [27]. In this study, we demonstrated that expression of Vitreoscilla hemoglobin significantly improves ARA and total lipid production in M. alpina.

Heterologous expression of $\mathrm{VHb}$ in M. alpina increased cell growth as well as oxygen uptake under non-limiting and limiting dO2 conditions. Furthermore, $\mathrm{VHb}$ expression shortened the lag phase for the growth of $M$. alpina and the appearance of ARA. A similar observation has previously been reported in $S$. occidentalis, in which alpha-amylase appears earlier in the culture medium of VHb-expressing cells [28], and the result is also consistent with the expression of $\mathrm{VHb}$ in Gluconobacter oxydans [29] and Phellinus igniarius [8]. Importantly, the morphology of the VHb-20 strain changed significantly. The mutant $\mathrm{VHb}-20$ stain formed small homogeneous globules, whereas the wild-type strain formed loose mycelia (Fig. 5). Previous experiments have shown that in late fermentation stages, the wild-type strain readily agglomerates, thus resulting in decreased dissolved oxygen. In contrast, mycelial pellets increased the fermentation fluidity, which is more conducive to dissolved oxygen and accumulation of lipids and ARA [7]. Moreover, the growth pattern of $M$. alpina has a large effect on ARA production [30]. These results are also consistent with findings by Higashiyama et al. [31] indicating that high concentrations of dissolved oxygen are necessary for obtaining smooth pellets, and the number of spherical fluffy pellets and the dissolved oxygen concentration are positively correlated.

Dissolved oxygen levels affect not only cell growth but also the synthesis of fatty acids in M. alpina. Therefore, the $\mathrm{VHb}-20$ and wild-type strains were analyzed for fatty acid and ARA content under normal culture conditions and dissolved oxygen-limiting conditions. Studies have shown that the expression of $\mathrm{VHb}$ increases the yield of ARA and total lipids, especially under oxygen-limiting conditions. It has been reported previously that $\mathrm{VHb}$ enhances ATP production and respiratory activity [32]. Evidence has shown that $\mathrm{VHb}$ improves the efficiency of oxidative phosphorylation of cells by regulating the activity of the relevant respiratory oxidase, thereby changing the central carbon metabolic pathway, and ultimately promoting cell growth and protein expression under hypoxic conditions [33, 34]. In addition, VHb also has peroxidase activity, and many heterologous hosts protect against oxidative invasion by expressing this gene, in agreement with the hypothesis that $\mathrm{VHb}$ is a carrier of oxygen in the cell $[12,35]$. On the basis of these observations, $\mathrm{VHb}$ expression may promote fatty acid synthesis and increase total lipid accumulation, owing to the enhancement of respiratory activity and ATP supply.

In addition, another prominent finding was that $\mathrm{VHb}$ expression significantly changed the fatty acid composition, particularly by decreasing the ratio of $\mathrm{C} 16: 0$ and C18:1 and increasing the ratio of C20:4 (ARA; Table 1). The biosynthesis of ARA strongly depends on oxygen accessibility, and some specific catalytic functions of 
various fatty acid desaturases and elongation enzymes involved in ARA synthesis in $M$. alpina have been elucidated [36, 37]. The oxygen requirement for desaturation was higher than that for cell growth and total lipid production. Therefore, the increase in ARA content in $\mathrm{VHb}-20$ may be a result of the overall increase of the fatty acid desaturase caused by the presence of heterologous hemoglobin. This speculation is consistent with the expression level of desaturases that we determined by qRT-PCR. It can be seen from Fig. 4 that four kinds of desaturases in the ARA metabolic pathway are $\Delta 9$-, $\Delta 12-, \Delta 6-, \Delta 5-$ desaturases respectively, and their expression levels are significantly up-regulated under both the aerobic and microaerobic conditions. Moreover, these desaturases were the key enzymes catalyzed the palmitic acid (C16: 0), stearic acid (C18: 0) and Oleic acid (C18: 1) converted to ARA. Therefore, we hypothesized that the increase of ARA production of $M$. alpina is probably due to the increase of the expression of desaturase and the improved desaturase activities in ARA metabolic pathway and the promotion of metabolic flow to ARA synthesis. More detailed mechanisms need further study.

\section{Conclusions}

In conclusion, the successful expression of $\mathrm{VHb}$ in $M$. alpina significantly increases ARA and lipid production as well as promotes cell growth and increase viability under both lower and normal dissolved oxygen conditions. Therefore, we suggest that expression of the $v g b$ gene in $M$. alpina might have good prospects in highdensity fermentation. However, the specific mechanisms underlying the effects of $\mathrm{VHb}$ in M. alpina are not clear and require further investigation.

\section{Additional file}

Additional file 1: (DOCX $263 \mathrm{~kb})$

\section{Abbreviations}

ARA: Arachidonic acid; ATMT: Agrobacterium tumefaciens-mediated transformation; CO: Carbon monoxide; DCW: Dry cell weight; DGLA: DihomoY-linolenic acid; DO: Dissolved oxygen; FAMEs: Fatty acid methyl esters; FID: Flame ionization detector; GLA: $y$-linolenic acid; HPH: hygromycinb phosphotransferase gene; IM: Inducing medium; LA: Linoleic acid; $M$. alpina: Mortierella alpina; MM: Minimal medium; PUFAs: Polyunsaturated fatty acids; VHb: Vitreoscilla hemoglobin

\section{Acknowledgements}

The authors are thankful to Prof. Yasuyuki Kubo for providing the Agrobacterium tumefaciens strain $\mathrm{C} 58 \mathrm{C} 1$ and plasmid pBIG4MRHrev. This study is a contribution to the international IMBER project.

\section{Funding}

This work is financially supported by the National Key Research and Development Program (no. 2016YFA0601400), the National Natural Science Foundation of China (no. 41306132), and the National High
Technology Research and Development Program of China (no. 2014AA021701). This study is a contribution to the international IMBER project.

\section{Availability of data and materials}

The strains, plasmids, primers, optimized vgb Sequence and Schematic representation of the plasmid construction are supplied as supporting information.

\section{Authors' contributions}

Huidan Zhang performed the experiments as part of her doctoral work. All this work was carried out under the supervision of Qiu Cui and Xiaojin Song. Xiaojin Song assisted in the bioreactor experiment. Qiu Cui and Yingang Feng also assisted in editing the manuscript. All the authors have read and approved the final manuscript.

\section{Competing interest}

The authors declare that they have no competing interests.

Ethics approval and consent to participate

Not applicable.

\section{Consent for publication}

Not applicable.

\section{Publisher's Note}

Springer Nature remains neutral with regard to jurisdictional claims in published maps and institutional affiliations.

\section{Author details}

'Shandong Provincial Key Laboratory of Energy Genetics, Qingdao Institute of Bioenergy and Bioprocess Technology, Chinese Academy of Sciences, No.189 Songling Road, Laoshan District, Qingdao, Shandong Province 266101, China. ${ }^{2}$ Key Laboratory of Biofuels, Qingdao Institute of Bioenergy and Bioprocess Technology, Chinese Academy of Sciences, Qingdao, Shandong 266101, China. ${ }^{3}$ Qingdao Engineering Laboratory of Single Cell Oil, Qingdao, Shandong 266101, China. ${ }^{4}$ University of Chinese Academy of Sciences, Beijing 100049, China.

Received: 6 June 2017 Accepted: 8 August 2017

Published online: 30 August 2017

\section{References}

1. You JY, Peng C, Liu X, Ji XJ, Lu J, Tong Q, et al. Enzymatic hydrolysis and extraction of arachidonic acid rich lipids from Mortierella alpina. Bioresour Technol. 2011;102:6088-94.

2. Wang HC, Yang B, Hao GF, Feng Y, Chen HQ, Feng L, et al. Biochemical characterization of the tetrahydrobiopterin synthesis pathway in the oleaginous fungus Mortierella alpina. Microbiology. 2011;157:3059-70.

3. Dedyukhina EG, Chistyakova TI, Vainshtein MB. Biosynthesis of arachidonic acid by micromycetes (review). Appl Biochem Microbiol. 2011:47:109-17.

4. Nie ZK, Ji XJ, Shang JS, Zhang AH, Ren LJ, Huang H. Arachidonic acid-rich oil production by Mortierella alpina with different gas distributors. Bioprocess Biosyst Eng. 2014;37:1127-32.

5. Wu WJ, Yan JC, Ji XJ, Zhang X, Shang JS, Sun LN, et al. Lipid characterization of an arachidonic acid-rich oil producing fungus Mortierella alpina. Chin J Chem Eng. 2015;23:1183-7.

6. Dedyukhina EG, Chistyakova TI, Mironov AA, Kamzolova SV, Minkevich IG, Vainshtein MB. The effect of $\mathrm{pH}$, aeration, and temperature on arachidonic acid synthesis by Mortierella alpina. Appl Biochem Microbiol. 2015;51:242-8.

7. Higashiyama K, Fujikawa S, Park EY, Okabe M. Image analysis of morphological change during arachidonic acid production by Mortierella alpina 1S-4. J Biosci Bioeng. 1999;87:489-94.

8. Zhu H, Sun S, Zhang S. Enhanced production of total flavones and exopolysaccharides via Vitreoscilla hemoglobin biosynthesis in Phellinus igniarius. Bioresour Technol. 2011;102:1747-51.

9. Li M, Wu J, Lin J, Wei D. Expression of Vitreoscilla hemoglobin enhances cell growth and Dihydroxyacetone production in Gluconobacter oxydans. Curr Microbiol. 2010:61:370-5.

10. Suen YL, Tang H, Huang J, Chen F. Enhanced production of fatty acids and astaxanthin in Aurantiochytrium sp. by the expression of Vitreoscilla hemoglobin. J Agric Food Chem. 2014;62:12392-8. 
11. Tao L, Sedkova N, Yao H, Ye RW, Sharpe PL, Cheng Q. Expression of bacterial hemoglobin genes to improve astaxanthin production in a methanotrophic bacterium Methylomonas sp. Appl Microbiol Biotechnol. 2006;74:625-33.

12. Stark BC, Pagilla KR, Dikshit KL. Recent applications of Vitreoscilla hemoglobin technology in bioproduct synthesis and bioremediation. App Microbiol Biotechnol. 2015;99:1627-36.

13. Frey $A D$, Kallio PT. Bacterial hemoglobins and flavohemoglobins: versatile proteins and their impact on microbiology and biotechnology. FEMS Microbiol Rev. 2003;27:525-45.

14. Zhang S, Wang J, Wei Y, Tang Q, Ali MK, He J. Heterologous expression of $\mathrm{VHb}$ can improve the yield and quality of biocontrol fungus Paecilomyces lilacinus, during submerged fermentation. J Biotechnol. 2014;187:147-53.

15. Bhave SL, Chattoo BB. Expression ofvitreoscilla hemoglobin improves growth and levels of extracellular enzyme in Yarrowia lipolytica. Biotechnol Bioeng. 2003;84:658-66.

16. Wang $X$, Sun $Y$, Shen $X$, Ke F, Zhao H, Liu Y, et al. Intracellular expression of Vitreoscilla hemoglobin improves production of Yarrowia lipolytica lipase LIP2 in a recombinant Pichia pastoris. Enzym Microb Technol. 2012;50:22-8.

17. Hao G, Chen H, Gu Z, Zhang H, Chen W, Chen YQ, Cullen D. Metabolic engineering of Mortierella alpina for enhanced Arachidonic acid production through the NADPH-supplying strategy. Appl Environ Microbiol. 2016;82(11):3280-8.

18. Takeno S, Sakuradani E, Tomi A, Inohara-Ochiai M, Kawashima H, Ashikari T, Shimizu S. Improvement of the fatty acid composition of an oil-producing filamentous fungus, Mortierella alpina 1S-4, through RNA interference with delta12-desaturase gene expression. Appl Environ Microbiol. 2005;71(9):5124-8.

19. Ando A, Sumida $Y$, Negoro H, Suroto DA, Ogawa J, Sakuradani E, et al. Establishment of Agrobacterium tumefaciens-mediated transformation of an oleaginous fungus, Mortierella alpina 1S-4, and its application for eicosapentaenoic acid producer breeding. Appl Environ Microbiol. 2009;75:5529-35.

20. Mackenzie D, Wongwathanarat $\mathrm{P}$, Carte A, Archer D. Isolation and use of a homologous Histone $\mathrm{H} 4$ promoter and a ribosomal DNA region in a transformation vector for the oil-producing fungus Mortierella alpina. Appl Environ Microbiol. 2000;66:4655-61.

21. Ando A, Sakuradani E, Horinaka K, Ogawa J, Shimizu S. Transformation of an oleaginous zygomycete Mortierella alpina 1S-4 with the carboxin resistance gene conferred by mutation of the iron-sulfur subunit of succinate dehydrogenase. Curr Genet. 2009;55:349-56.

22. Hao G, Chen H, Wang L, Gu Z, Song Y, Zhang H, et al. Role of malic enzyme during fatty acid synthesis in the oleaginous fungus Mortierella alpina. Appl Environ Microbiol. 2014;80:2672-8.

23. Takeno S, Sakuradani E, Tomi A, Inohara-Ochiai M, Kawashima H, Shimizu S. Transformation of oil-producing fungus, Mortierella alpina 1S-4, using Zeocin, and application to arachidonic acid production. J Biosci Bioeng. 2005;100:617-22.

24. Cheng YR, Sun ZJ, Cui GZ, Song XJ, Cui Q. A new strategy for strain improvement of Aurantiochytrium sp. based on heavy-ions mutagenesis and synergistic effects of cold stress and inhibitors of enoyl-ACP reductase. Enzym Microb Technol. 2016;93-94:182-90.

25. Kikukawa H, Sakuradani E, Ando A, Okuda T, Ochiai M, Shimizu S, et al. Disruption of lig4 improves gene targeting efficiency in the oleaginous fungus Mortierella alpina 1S-4. J Biotechnol. 2015;208:63-9.

26. Ji XJ, Zhang AH, Nie ZK, Wu WJ, Ren LJ, Huang H. Efficient arachidonic acidrich oil production by Mortierella alpina through a repeated fed-batch fermentation strategy. Bioresour Technol. 2014;170:356-60.

27. Li X, Lin Y, Chang M, Jin Q, Wang X. Efficient production of arachidonic acid by Mortierella alpina through integrating fed-batch culture with a two-stage pH control strategy. Bioresour Technol. 2015;181:275-82.

28. Suthar $\mathrm{DH}$, Chattoo BB. Expression of Vitreoscilla hemoglobin enhances growth and levels of alpha-amylase in Schwanniomyces occidentalis. Appl Microbiol Biotechnol. 2006;72:94-102.

29. Li MH, Wu JA, Lin JP, Wei DZ. Expression of Vitreoscilla hemoglobin enhances cell growth and Dihydroxyacetone production in Gluconobacter oxydans. Curr Microbiol. 2010;61:370-5.

30. Koike Y, Cai HJ, Higashiyama K, Fujikawa S, Park EY. Effect of consumed carbon to nitrogen ratio of mycelial morphology and arachidonic acid production in cultures of Mortierella alpina. J Biosci Bioeng. 2001;91:382-9.
31. Higashiyama KM, K Tsujimura $\mathrm{H}$. Effects of dissolved oxygen on the morphology of an Arachidonic acid production by Mortierella alpina 1S-4. Biotechnol Bioeng. 1998;63:442-8.

32. Kallio PT, Kim DJ, Tsai PS, Bailey JE. Intracellular expression of Vitreoscilla hemoglobin alters Escherichia-Coli energy-metabolism under oxygenlimited conditions. Eur J Biochem. 1994;219:201-8.

33. Ramandeep HKW, Raje M, Kim KJ, Stark BC, Dikshit KL, et al. Vitreoscilla hemoglobin - intracellular localization and binding to membranes. J Biol Chem. 2001;276:24781-9.

34. Zhang L, Li Y, Wang Z, Xia Y, Chen W, Tang K. Recent developments and future prospects of Vitreoscilla hemoglobin application in metabolic engineering. Biotechnol Adv. 2007;25:123-36.

35. Li W, Zhang Y, Xu H, Wu L, Cao Y, Zhao H, et al. pH-induced quaternary assembly of Vitreoscilla hemoglobin: the monomer exhibits better peroxidase activity. Biochim Biophys Acta (BBA) - Proteins and Proteomics. 2013;1834:2124-32.

36. Sakuradani E. Advances in the production of various polyunsaturated fatty acids through oleaginous fungus Mortierella alpina breeding. Biosci Biotechnol Biochem. 2010;74:908-17.

37. Vongsangnak W, Ruenwai R, Tang X, Hu X, Zhang H, Shen B, et al. Genomescale analysis of the metabolic networks of oleaginous Zygomycete fungi. Gene. 2013:521:180-90.

\section{Submit your next manuscript to BioMed Central and we will help you at every step:}

- We accept pre-submission inquiries

- Our selector tool helps you to find the most relevant journal

- We provide round the clock customer support

- Convenient online submission

- Thorough peer review

- Inclusion in PubMed and all major indexing services

- Maximum visibility for your research

Submit your manuscript at www.biomedcentral.com/submit
) Biomed Central 